\title{
Aplicación del aprendizaje cooperativo a través de Tumblr para la adquisición de competencias
}

\section{Application of cooperative learning through Tumblr for the acquisition of competencies}

\author{
Manuel Toledo Padrón ${ }^{1}$, Antonio José Cuesta Vázquez², José Ángel Siles \\ López $^{3}$ \& María de los Ángeles Martín Santos ${ }^{4}$
}

Fecha de recepción: 21/04/2020; Fecha de revisión: 16/07/2020; Fecha de aceptación: 24/09/2020

Cómo citar este artículo:

Toledo, M, Cuesta, A.J., Siles, J.A. \& Martín, M.A. (2019). Aplicación del aprendizaje cooperativo a través de Tumblr para la adquisición de competencias. Revista de Innovación y Buenas Prácticas Docentes, 9(2), 123-136.

Autor de Correspondencia: iq2masam@uco.es

\section{Resumen:}

El potencial de las redes sociales ha supuesto la aparición de herramientas alternativas para la adquisición de competencias a través de dinámicas de trabajo en grupo e interacción social. El objetivo de este proyecto es que el alumnado construya su propio aprendizaje y se enriquezca del intercambio de ideas y la cooperación con sus compañeros/as. El alumnado fue dividido en grupos de 5-7 componentes y se llevó a cabo una sesión introductoria en la creación de un microblog y de planificación de actividades a través de la plataforma Tumblr. Entre los resultados más destacados, se observó una tasa de éxito (91\%) y rendimiento (66\%) más elevada que en la anualidad previa. Los grupos con publicaciones de calidad y rigor científico mostraron una mayor tasa de éxito que los grupos con un elevado número de publicaciones menos selectivas. El número de aprobados con un rango de 5,0 a 7,5 se vio incrementado, lo que se traduce en una mejora en la adquisición de competencias y conocimientos. Por lo tanto, esta herramienta educativa innovadora permite al alumnado generar interdependencia positiva; promover valores como la responsabilidad, comunicación y trabajo en equipo; mejorar las relaciones interpersonales y habilidades sociales; y construir conocimiento en sociedad.

Palabras clave: aprendizaje en grupo, evaluación formativa, proyecto piloto, tecnologías de la información y de la comunicación

\begin{abstract}
: contributes to build knowledge in society. project

\footnotetext{
Universidad de Córdoba (España), z22topam@uco.es; 0000-0001-8160-5076

3 Universidad de Córdoba (España), a92siloj@uco.es; 0000-0002-9546-083X

${ }^{4}$ Universidad de Córdoba (España), iq2masam@uco.es; 0000-0002-7184-9816
}

2 Universidad de Córdoba (España), ajcuesta@uco.es; 0000-0002-4153-9470
\end{abstract}

The potential of social networks has led to the appearance of alternative tools for the acquisition of competences through group work dynamics and social interaction. The aim of this project is for students to build their own learning and enrich themselves through the exchange of ideas and cooperation with their peers. The students were divided into groups of 5-7 components and an introductory session on creating a microblog and activity planning through the Tumblr platform was carried out. Among the most relevant results, a higher success rate $(91 \%)$ and performance $(66 \%)$ was observed compared to previous years. The groups with quality publications and scientific rigor showed a higher success rate than the groups with a high number of less selective publications. The number of students who passed with a score from 5.0 to 7.5 increased, which translates into an improvement in the acquisition of skills and knowledge. Therefore, this innovative educational tool allows students to generate positive interdependence; promote values such as responsibility, communication and teamwork; improve interpersonal relationships and social skills; and

Key Words: formative assessment, group learning, information and communication technologies, pilot 


\section{INTRODUCCIÓN}

Bajo el paraguas de las redes sociales, se distinguen diversas plataformas cuyo enfoque principal es proporcionar medios para que las personas se conecten e interactúen, mientras que otra corriente se caracteriza por plataformas donde los contenidos son generados por los propios usuarios. En ambos casos su finalidad se centra en la producción de material creativo (van Dijck, 2013). En este entorno, de forma bidireccional, Tumblr se clasifica como una plataforma de microblogging que ofrece características tanto de interacción de usuarios, como de producción de contenidos propios, con ambas dimensiones igualmente enfatizadas (Chang, Tang, Inagaki \& Liu, 2014). Su funcionalidad es dinámica y se basa en compartir públicamente contenido en forma de imágenes, vídeos, enlaces y textos en un formato reducido, lo que se conoce como microblogueo (microblogging, en inglés). Tumblr fue fundada en 2007 por David Karp y adquirida por Yahoo en junio de 2013, debido a su popularidad. Actualmente, Tumblr tiene 492 millones de blogs y más de 150 mil millones de publicaciones (Tumblr, 2020). Además, es una plataforma accesible en 18 idiomas y posee más de 475 millones de cuentas de usuarios.

La estrategia para hacer llegar su contenido a otros/as usuarios/as que visiten esta plataforma (sin necesidad de un registro previo) resulta ser un formato intermedio entre un blog, es decir una página web donde se suelen compartir contenidos en forma de entradas desarrolladas con todo detalle, y el formato de la red social Twitter, en la que los contenidos que se comparten están limitados a 160 caracteres. De esta forma, el enfoque está dirigido a un/a usuario/a que desea ver contenido relacionado con una temática y pueda encontrar rápidamente numerosas entradas a la vez, mostrándose algunos detalles del contenido sin la necesidad de entrar en cada una de ellas.

En el ámbito educativo, este tipo de plataformas favorecen la comunicación entre el alumnado y entre éste y el profesor, facilitando la evaluación continua, al mismo tiempo que despiertan en el estudiantado su capacidad comunicativa y de trabajo en equipo, sin olvidar la posibilidad de mantener contacto con profesionales y expertos relacionados con el temario académico (Imbernón, Silva \& Guzmán, 2011). De esta forma se hace posible materializar el aprendizaje constructivista, colaborativo y por competencias, tanto dentro como fuera del aula, a lo que se suman el protagonismo del alumnado frente al del profesorado, la bidireccionalidad ante la unidireccionalidad y, en definitiva, otros planteamientos didácticos que consideran al estudiante un agente activo (Rubio-Millares, 2019).

En este contexto, el Proyecto de Innovación Docente "Aplicación del aprendizaje cooperativo a través de Tumblr para la adquisición de competencias" aprovecha las ventajas que ofrece esta plataforma, así como su facilidad de uso y popularidad de las redes sociales en general, con el objetivo de fomentar el aprendizaje colaborativo con un enfoque didáctico. Sin embargo, no es la primera vez que las redes sociales se usan para potenciar unos objetivos en educación. Existen experiencias previas en las que las redes sociales se ponen a prueba para comprobar su influencia en el desarrollo de habilidades, como pueden ser la comunicación o la conciencia social (De Haro, 2010), y es en estas experiencias previas en las que se puede basar el análisis. De hecho, en el contexto de la educación superior, existen numerosos trabajos que estudian el uso educativo de las redes sociales en el alumnado universitario y sus actitudes cuando se persigue un uso educativo de las mismas (Martínez, Fortuño \& Vidal, 2016; Martínez, 2014; Gómez, Roses \& Farias, 2012). 
Es en este sentido que el Proyecto de Innovación Docente va más allá del uso de Tumblr para la adquisición de competencias básicas como la expresión oral y escrita, o la mejora en las habilidades de comunicación (Yunus \& Salehi, 2012). Se fomenta su uso desde el marco de la asignatura Tratamiento de Aguas Residuales en el título de Ciencias Ambientales ( $3^{\text {er }}$ curso) de la Universidad de Córdoba y se persigue un desarrollo de competencias específicas, como la mejora en la comprensión de los conceptos tecnológicos y científicos trabajados en la asignatura, así como su relación con otros materiales extra-académicos que se pueden encontrar en los distintos medios, incluyendo Internet. Se ha postulado de especial interés la comprensión de información científica sobre la temática y su correcta divulgación: "No solo es saber, sino hacerse entender".

Desde este enfoque, se han realizado subgrupos de alumnos/as matriculados/as en la asignatura durante el curso 2019/20 y se les ha dado de alta en la plataforma. Cada grupo debe operar con una puesta en común interna antes de cada publicación y se pretende fomentar las interacciones entre grupos a través de la plataforma. Así se logra una mejora de las competencias desarrolladas en la asignatura en la que los conceptos se ejemplifican con materiales compartidos por y entre el propio alumnado, relevantes en el contexto científico-social que les rodea (por ejemplo, trabajando materiales relacionados con la investigación publicada por fuentes científicas reputadas como Centros tecnológicos, CSIC, grupos de investigación de universidades y sector empresarial gestor de aguas residuales, en resumen, usando contenido relevante de la actualidad informativa científica y tecnológica). Además, con ayuda de la plataforma Tumblr se aúnan estos contenidos bajo una serie de etiquetas comunes que permiten y fomentan la discusión de estos contenidos (Bourlai, 2018). Por último, es posible medir el grado de aprovechamiento de las competencias desarrolladas, incluyéndolas en las pruebas de evaluación de la asignatura a través de un cuestionario simple generado a partir de la supervisión del profesorado (visualización de las publicaciones y comentarios del alumnado grupo de estudio), de forma que estas experiencias no queden completamente desconectadas de los objetivos de adquisición de las competencias de la asignatura.

\section{DESARROLLO DE LA EXPERIENCIA DE INNOVACIÓN}

\subsection{Competencias desarrolladas}

Con el enfoque de planificar e implementar estrategias, metodologías, medios y acciones necesarias para la adquisición de competencias y conocimientos por parte del alumnado, surge el aprendizaje colaborativo a través de dinámicas de trabajo en grupo e interacción social. Para adquirir y desarrollar competencias específicas de la asignatura Tratamiento de Aguas Residuales del Grado de Ciencias Ambientales de un modo flexible y alternativo mediante el uso de la plataforma Tumblr se ha utilizado la interacción entre iguales autoalimentada en la plataforma por los grupos de alumnos/as. Como unidad metodológica, el subgrupo de alumnos/as, trabajan competencias de liderazgo y trabajo colaborativo. Aunque teniendo en cuenta la guía docente de la asignatura (TAR, 2019), se han observado tres competencias que el proyecto ha desarrollado con resultados prometedores:

1. Conocer y perfeccionar el nivel de usuario en el ámbito de las TIC (CU2).

2. Ser capaz de gestionar la información (CB3) y razonamiento crítico (CB12). 
3. Capacidad de análisis multidisciplinar de datos, índices e indicadores ambientales y cuantitativos (CE3).

\subsection{Objetivos}

A cada una de las competencias se atribuyen diversos objetivos específicos y actividades que el alumnado debía completar con diversas metodologías que se han trabajado, alcanzando las metas previstas con la implementación del proyecto en la asignatura; siempre bajo la supervisión del profesorado responsable de la misma. Los objetivos, tareas, actividades y recursos asociados a cada competencia se desglosan en la Figura 1.

\begin{tabular}{|c|c|c|c|}
\hline Competencia & Objetivo & Actividad & $\begin{array}{c}\text { Recursos } \\
\text { asociados }\end{array}$ \\
\hline $\begin{array}{l}\text { 1: Conocer y } \\
\text { perfeccionar el } \\
\text { nivel de usuario en } \\
\text { el ámbito de las } \\
\text { TIC (CU2). }\end{array}$ & $\begin{array}{l}\text { 1.1: Usar de forma autónoma } \\
\text { una herramienta de } \\
\text { microblogging para el desarrollo } \\
\text { del aprendizaje cooperativo. }\end{array}$ & $\begin{array}{l}\text { 1.1.1: Taller para } \\
\text { el aprendizaje y } \\
\text { utilización de } \\
\text { Tumblr. }\end{array}$ & \multirow{5}{*}{$\begin{array}{c}\text { Ordenador, } \\
\text { puntero, } \\
\text { conexión a } \\
\text { internet, } \\
\text { aplicación } \\
\text { Tumblr, } \\
\text { tutorial, trabajo } \\
\text { autónomo del } \\
\text { alumno/a, } \\
\text { actualización y } \\
\text { mejora de } \\
\text { software. }\end{array}$} \\
\hline \multirow{3}{*}{$\begin{array}{l}\text { 2: Ser capaz de } \\
\text { gestionar la } \\
\text { información (CB3) y } \\
\text { razonamiento } \\
\text { crítico (CB12). }\end{array}$} & $\begin{array}{l}\text { 2.1: Capacitar al alumnado en la } \\
\text { búsqueda de información } \\
\text { relevante con respecto a la }\end{array}$ & $\begin{array}{l}\text { 2.1.1: Búsqueda } \\
\text { de noticias } \\
\text { divulgativas. }\end{array}$ & \\
\hline & $\begin{array}{l}\text { problemática asociada a las } \\
\text { aguas residuales y su } \\
\text { tratamiento mediante diferentes } \\
\text { fuentes científicas y de } \\
\text { divulgación. }\end{array}$ & $\begin{array}{l}\text { 2.1.2: Búsqueda } \\
\text { de información } \\
\text { científico-técnica }\end{array}$ & \\
\hline & $\begin{array}{l}\text { 2.2: Desarrollar la capacidad del } \\
\text { alumnado para discriminar de } \\
\text { forma crítica la calidad y } \\
\text { fiabilidad de la fuente de } \\
\text { información disponible en la red. }\end{array}$ & $\begin{array}{l}\text { 2.2.1: Cribado de } \\
\text { información }\end{array}$ & \\
\hline $\begin{array}{l}\text { 3: Capacidad de } \\
\text { análisis } \\
\text { multidisciplinar de } \\
\text { datos, índices e } \\
\text { indicadores } \\
\text { ambientales y } \\
\text { cuantitativos (CE3). }\end{array}$ & $\begin{array}{l}\text { 3.1: Correlacionar datos } \\
\text { analíticos e indicadores de } \\
\text { calidad para conocer la } \\
\text { idoneidad del tratamiento } \\
\text { aplicado a las aguas residuales } \\
\text { para su vertido a la cuenca } \\
\text { receptora, teniendo en cuenta } \\
\text { las características que regulen } \\
\text { dichas cuencas hidrográficas. }\end{array}$ & $\begin{array}{l}\text { 3.1.1: Análisis de } \\
\text { datos }\end{array}$ & \\
\hline
\end{tabular}

Figura 1. Competencias, objetivos, actividades y recursos del proyecto Tumblr. Fuente: Elaboración propia

\subsection{Materiales y métodos}

\subsubsection{Plataforma Tumblr}

Para utilizar Tumblr solo es necesario registrarse en la web "www.tumblr.com" y elegir un nombre de usuario y contraseña. La membresía de Tumblr es gratuita y una vez completado el registro se podrá personalizar la visualización del blog de usuario, colocando un título, completando el perfil, eligiendo la plantilla de visualización del diseño web, el formato de imágenes y todo lo relacionado a la parte visual de este espacio para hacerlo atractivo a los usuarios de todo el mundo. Los usuarios no sólo pueden crear sus propias publicaciones de blog, como en un sitio de blogs tradicional, sino que también pueden "seguir" otros blogs. Este sistema de seguimiento es parte de lo que hace que Tumblr sea más una comunidad que una colección de publicaciones de blog personales separadas con audiencias restringidas. 
Registrándose para crear un blog (o varios blogs) un usuario tiene la opción de siete diferentes tipos de publicaciones según la catalogación de contenido compartido: texto, foto, cita, enlace, chat, audio y video. La Figura 2 muestra la barra de herramientas desde la cual los usuarios seleccionan el tipo de publicación que desean usar y añadir al blog.

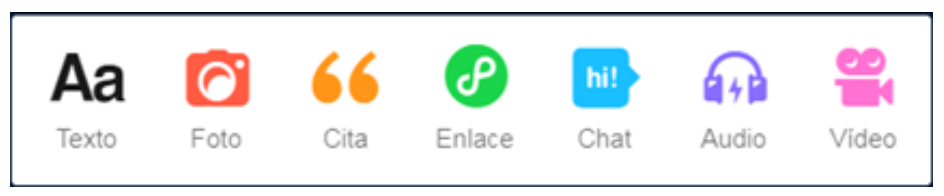

Figura 2. Barra de herramientas de Tumblr. Fuente: http://www.tumblr.com/

Otro tipo de publicación se crea cuando el propietario de un blog responde públicamente a un mensaje recibido por otro usuario. Los usuarios pueden visitar blogs individualmente para ver todas sus publicaciones, o seguirlos para visualizar sus publicaciones más recientes en una página central llamada "Tablero" (similar a la línea de tiempo de Twitter). Los usuarios también tienen la opción de buscar publicaciones individuales, incluyendo palabras clave específicas o etiquetas. Una vez que un usuario sigue un blog, todas las publicaciones del mismo aparecen en el "Tablero" del usuario (similar a una página de inicio), en orden cronológico inverso. El tablero de instrumentos, que se muestra en la Figura 3, es la interfaz de actividad principal, desde la cual los usuarios ven, interactúan y crean publicaciones. Los tipos de publicaciones más comunes tienden a ser Foto y Texto (Chang et al., 2014).

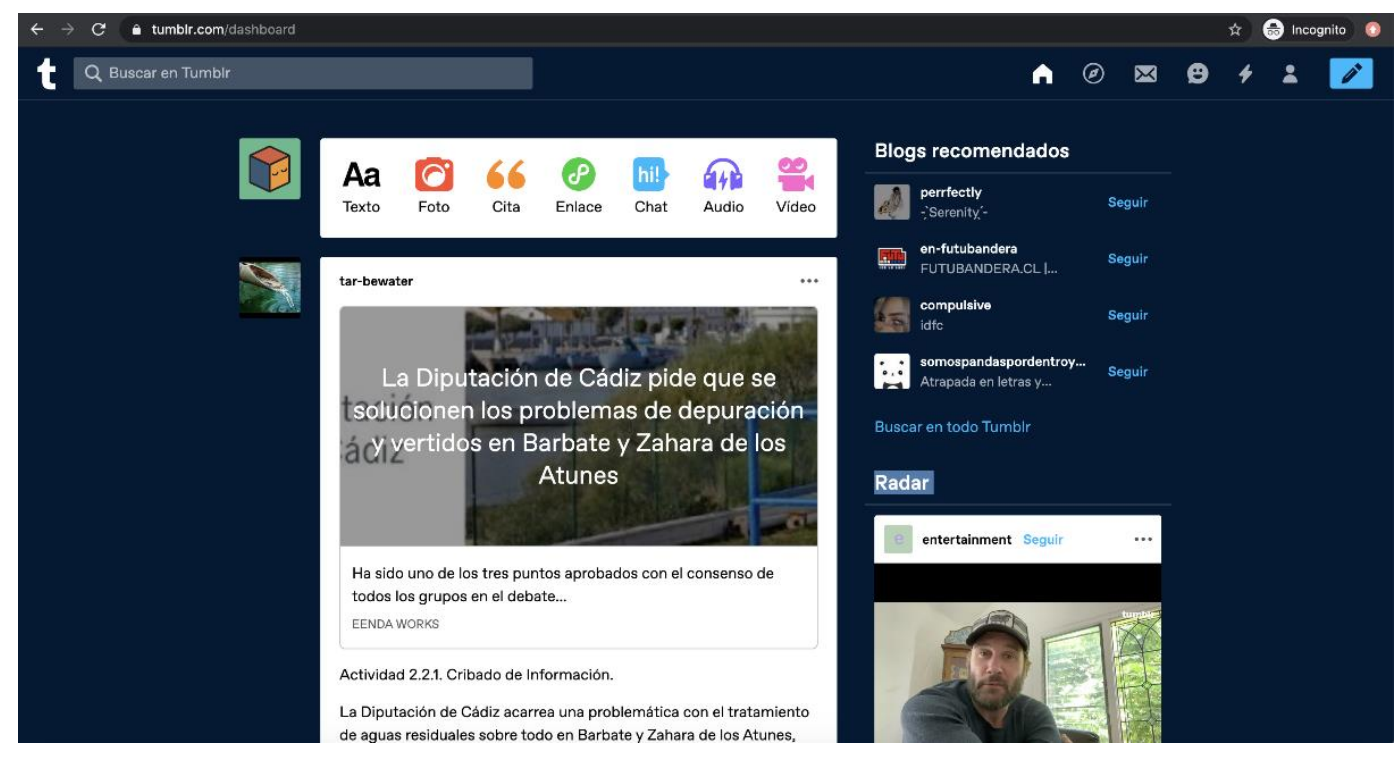

Figura 3. Tablero - Interfaz de actividad principal de Tumblr. Fuente: http://www.tumblr.com/

Al desplazarse hacia abajo en el tablero, los usuarios pueden ver todas las publicaciones de los blogs que siguen, así como sus propias publicaciones. Al hacer clic en "Me gusta", una publicación se guarda en un archivo personal al que se puede acceder más tarde, mientras se llevan a cabo otras publicaciones en su propio blog. De esta manera, cada vez que se hace una publicación, recibe una exposición exponencial. Si un usuario sigue una gran cantidad de blogs, es probable que vea las mismas 
publicaciones populares aparecer varias veces en su panel de control. Además existe la opción del "Reblogging" que se da cuando un usuario publica una publicación hecha por otro usuario y la fuente de la publicación original se mantiene visible como un enlace en el que se puede hacer clic en la parte inferior de la publicación. En este sentido, Tumblr desaconseja publicar sin la fuente original (Deller, 2015), lo cual aporta una mayor validez y fiabilidad de la información. Otros estudios, han observado que Tumblr, con su amplia estructura de red, es una comunidad más unida en comparación con otros sitios web de blogs (Bourlai, 2018).

\subsubsection{Metodología empleada}

El alumnado matriculado en la asignatura Tratamiento de Aguas Residuales del Grado de Ciencias Ambientales se ha dividido en grupos de 5-7 componentes, agrupados en libre disposición para evitar que la agrupación forzada tensione los grupos de trabajo y los roles adoptados por sus componentes, asociando a cada grupo un nombre de usuario (iniciales de la asignatura "TAR") y una identificación relativa al número de grupo correspondiente (por ejemplo: TAR1, TAR2, TAR3, etc.).

Una vez organizados los grupos, se ha llevado a cabo una sesión teórico-práctica por parte del profesorado, con fines didácticos de iniciación en la creación de un microblog mediante el uso de la plataforma Tumblr, con ayuda de una presentación. Asimismo, se ha planificado el desarrollo de las actividades para la puesta en práctica de esta plataforma de aprendizaje cooperativo, analizando los objetivos y actividades a realizar por todos los grupos.

Durante la primera semana, posterior a la sesión teórico-práctica, los/as alumnos/as realizaron el registro de los componentes del grupo y crearon el blog grupal. Una vez creados los blogs, el alumnado facilitó los usuarios y contraseñas a los profesores responsables de la asignatura para así poder llevar a cabo un seguimiento de las publicaciones y comentarios realizados en los blogs. Además, durante este tiempo, pudieron publicar alguna noticia e hicieron uso del "Reblogging", entre otras funcionalidades, para dar constancia del aprendizaje y la utilización de la plataforma Tumblr, cumpliendo así con el objetivo 1.1.

Unas semanas después, los profesores responsables observaron, mediante las noticias e información científico-técnica publicadas por los grupos, que era necesario una sesión guiada con el alumnado para aclarar las dudas y focalizar el trabajo realizado por los/as mismos/as con el fin de mejorar el uso de la plataforma a lo largo del desarrollo del proyecto. En este sentido, no solo era necesario que publicaran información relevante relacionada con la asignatura, sino que era imprescindible desarrollar un aprendizaje colaborativo, donde hubiera una retroalimentación de la información publicada por los distintos grupos, así como de desarrollar el análisis de los datos publicados y correlacionarlos con la legislación actual, identificando los indicadores de calidad e idoneidad de las tecnologías y fundamentos científico-prácticos desarrollados en el temario de la asignatura, de acuerdo con los objetivos 2.1, 2.2 y 3.1. Estas estrategias de feedback tienen una efectividad demostrada en este tipo de actividades de evaluación formativa (Santana, Jiménez, Pintor \& Hernández, 2017), permitiéndoles detectar, desde un criterio ético, los errores y puntos débiles de la información publicada por los distintos grupos. Además, se enfatizó la importancia de reflejar, en cada una de las publicaciones, el tipo de actividad con la que estaba relacionada (búsqueda de noticias divulgativas y de información científico-técnica, cribado de información y análisis de datos) y, de este modo, facilitar al profesorado responsable la evaluación de las publicaciones.

Una vez finalizado el temario de la asignatura y las publicaciones relativas a la misma, el profesorado responsable llevó a cabo una evaluación al alumnado mediante un examen escrito con una serie de preguntas relacionadas con las noticias científicas publicadas en Tumblr por los distintos grupos. Las preguntas debían ser estructuradas 
y concisas, pero además siguiendo distintas taxonomías de enseñanza-aprendizaje. Un total de 5 preguntas fueron seleccionadas, las cuales se describen en la Figura 4.

\begin{tabular}{cl}
\hline Pregunta & \multicolumn{1}{c}{ Descripción } \\
\hline 1 & $\begin{array}{l}\text { ¿En qué consiste la electrocoagulación? Señala alguna ventaja destacable } \\
\text { respecto a los procesos convencionales en el tratamiento de aguas } \\
\text { residuales. }\end{array}$ \\
\hline 2 & $\begin{array}{l}\text { ¿En qué ayuda a la técnica de floculación la utilización sensores durante } \\
\text { el tratamiento de las aguas residuales? }\end{array}$ \\
\hline 3 & $\begin{array}{l}\text { ¿En qué consiste el proceso de tratamiento de aguas residuales mediante } \\
\text { el consorcio algas-bacterias? Y ¿qué ventajas puede tener para un } \\
\text { planteamiento sostenible del tratamiento de aguas residuales? }\end{array}$ \\
\hline 4 & $\begin{array}{l}\text { ¿Qué utilidad pueden tener los lodos del tratamiento de aguas residuales? } \\
\text { (hencione dos beneficios que puede tener el uso de plantas acuáticas }\end{array}$ \\
\hline 5 &
\end{tabular}

Figura 4. Preguntas de evaluación del proyecto Tumblr.

Fuente: Elaboración propia

\section{RESULTADOS}

La evaluación del proyecto se ha realizado en dos etapas. Por una parte, mediante la evaluación continua, en la que los profesores responsables del proyecto han analizado de forma quincenal los avances y actividad de los grupos del alumnado. Estos avances incluyen tanto las nuevas contribuciones aportadas en la red social, como las interacciones entre ellos en forma de comentarios y difusión de materiales. Por otra parte, mediante la evaluación final, ya que el alumnado tuvo que responder en el examen final a una serie de preguntas relacionadas con sus contribuciones aportadas.

Los resultados obtenidos en el desarrollo del presente proyecto de innovación docente se han analizado en función de los objetivos específicos atribuidos a cada una de las competencias.

El objetivo 1.1. (Usar de forma autónoma una herramienta de microblogging), cuyo cumplimiento está asociado a la actividad 1.1.1 (Taller para el aprendizaje y utilización de Tumblr), se evaluó "la creación y destreza en el desarrollo del miniblog y los grupos de trabajo", se considera que tuvo un grado de cumplimiento muy alto. No solamente la mayoría de los grupos se prestaron y se dieron de alta dentro del plazo establecido, sino que por iniciativa propia los equipos pusieron su primera publicación en la plataforma. Tan solo un grupo, al no asistir a las clases de la asignatura, no completó la actividad a tiempo, aunque sí logró el objetivo con posterioridad al plazo inicial establecido.

El objetivo 2.1. (Capacitar al alumnado en la búsqueda de información relevante con respecto a la problemática asociada a las aguas residuales mediante diferentes fuentes como las científicas y de divulgación), estaba asociado a las actividades 2.1.1 (Búsqueda de noticias divulgativas) y 2.1.2 (Búsqueda de información técnica). Ambas actividades fueron evaluadas periódicamente, con una frecuencia quincenal, y el alumnado de nuevo mostró un elevado cumplimiento del objetivo establecido. Si bien se observaron diversos grados de participación, desde el mínimo imprescindible (una publicación cada dos semanas), hasta grados altos de participación (varias publicaciones por semana), el seguimiento continuo del blog por parte de los profesores participantes en el proyecto permitió un alto cumplimiento del objetivo establecido.

El objetivo 2.2. (Desarrollar la capacidad del alumnado para discriminar de forma crítica la calidad y fiabilidad de la fuente de información disponible en la red), asociado a la actividad 2.2.1 (Cribado de información), presentó un grado más dispar de 
cumplimiento. Por regla general, la mayoría de los grupos lograron encontrar fuentes fiables de información, pero les resultó más complicado encontrar fuentes cuya información fuese incompleta o incluso incorrecta, para poder comprobar su veracidad y exactitud en base a los conocimientos adquiridos en la asignatura. En este sentido hubiese sido interesante facilitar una colección de documentos, sin revelar la fuente, para potenciar el análisis crítico de los contenidos sin basar sus conclusiones en sesgos como el principio de autoridad.

Por último, el objetivo 3.1. (Correlacionar datos analíticos e indicadores de calidad para conocer la idoneidad del tratamiento aplicado a las aguas residuales para su vertido a la cuenca receptora, teniendo en cuenta las características que regulen dichas cuencas hidrográficas), asociado a la actividad 3.1.1., resultó ser el más interesante para la consecución de objetivos y resultados de aprendizaje de la asignatura, ya que en ella pudieron practicar con datos reales y comprobar si se cumplen los estándares fijados (límites de vertido de aguas residuales, normativa del tratamiento de lodos de EDAR, emisiones odoríferas, etc.) y los niveles de calidad de los tratamientos estudiados en la asignatura.

Para evaluar el efecto que ha tenido la inclusión de esta actividad en el rendimiento del alumnado, es conveniente realizar un estudio comparativo entre dos años consecutivos, bajo la hipótesis de que el cambio más significativo en el desarrollo de las competencias de la asignatura es la inclusión de esta actividad. En la Tabla 1 se recogen los resultados del curso 2019/20 y el precedente 2018/19 en cuanto a alumnos/as presentados/as y aprobados/as.

Tabla 1.

Resultados académicos. Cursos 2018/2019 y 2019/2020.

\begin{tabular}{lcc}
\hline & $\mathbf{2 0 1 8 / 2 0 1 9}$ & $\mathbf{2 0 1 9 / 2 0 2 0}$ \\
\hline Total alumnos/as matriculados/as & 50 & 32 \\
\hline Presentados/as & 36 & 23 \\
\hline No presentados/as & 13 & 9 \\
\hline № de aprobados & 31 & 21 \\
\hline № de suspensos & 5 & 2 \\
\hline Tasa de éxito (\%) & 86 & 91 \\
\hline Tasa de rendimiento (\%) & 62 & 66 \\
\hline
\end{tabular}

Fuente: Elaboración propia

Dado el número tan dispar de alumnos/as en ambas anualidades, debido al descenso que transitoriamente sufrió la titulación hace tres años, es conveniente analizar los datos en términos relativos. Se comparan los datos de la primera convocatoria pues es donde se encuentra el grueso de la evaluación de los/as alumnos/as. En la segunda convocatoria del curso 2018/19 superaron la asignatura dos de los cuatro alumnos presentados y en el 2019/20 uno de tres presentados. Con ello, se aprecia que no se modifican sustancialmente las tasas de éxito y rendimiento.

A partir de los datos obtenidos, se ha observado que la tasa de éxito ha sido mayor este curso (91\% frente a $86 \%$ ), al igual que la tasa de rendimiento ha sido ligeramente más elevada (66\% frente a $62 \%$ ). Como la asignatura incluye en su metodología y evaluación multitud de aspectos, es conveniente realizar un análisis de las actividades de este proyecto de innovación y evaluar si han podido influir en esta tendencia positiva. Experiencias previas en este sentido apuntan a que, si bien el alumnado suele mostrar una actitud positiva hacia el uso de redes sociales en el contexto académico, la utilidad pedagógica de las mismas es más cuestionable, principalmente por no utilizarse en este contexto (Vidal, Martínez, Fortuño \& Cervera, 2011). Para ello, en la Tabla 2 se muestra una comparativa entre los distintos grupos que han participado en la actividad, asociando la asistencia (como una medida del grado de interés en las actividades), a su rendimiento en la calificación final. 
Tabla 2.

Resultados académicos de la asignatura por grupos de trabajo Tumblr 2019/2020.

\begin{tabular}{|c|c|c|c|c|}
\hline \multirow[b]{2}{*}{$\begin{array}{c}\text { Grupo de trabajo } \\
\text { Tumblr }\end{array}$} & \multirow{2}{*}{$\begin{array}{c}\text { RELATIVO A LA } \\
\text { ASIGNATURA } \\
\text { Asistencia a las } \\
\text { sesiones } \\
\text { presenciales (\%) }\end{array}$} & \multicolumn{3}{|c|}{$\begin{array}{c}\text { RELATIVO AL GRUPO DE LA ACTIVIDAD } \\
\text { TUMBLR }\end{array}$} \\
\hline & & $\begin{array}{l}\text { Aprobados } \\
(\%)\end{array}$ & $\begin{array}{l}\text { Suspensos } \\
(\%)\end{array}$ & $\begin{array}{c}\text { No } \\
\text { presentados } \\
(\%)\end{array}$ \\
\hline 1 & 90 & 40 & 20 & 40 \\
\hline 2 & 67 & 50 & 0 & 50 \\
\hline 3 & 92 & 50 & 33 & 17 \\
\hline 4 & 50 & 84 & 0 & 16 \\
\hline 5 & 42 & 50 & 0 & 50 \\
\hline
\end{tabular}

Fuente: Elaboración propia

Se ha observado que la asistencia por sí misma no es un indicador fiable del rendimiento final del alumnado en la asignatura: los grupos 1 y 3 , con una mayor tasa de asistencia, tuvieron un rendimiento muy dispar de sus componentes, superando la asignatura aproximadamente la mitad de ellos. En cambio, en el grupo 4, con una menor tasa de asistencia, superaron casi en su totalidad la asignatura, solamente un/a alumno/a de este no obtuvo el aprobado por no presentarse al examen. La no cumplimentación de otras herramientas de evaluación ha conducido a gran parte de los resultados calificados como no presentados, pues no han llegado a testar sus conocimientos frente al examen en primera convocatoria. En cuanto a los grupos 2 y 5 , se observa que la mitad de sus componentes consiguió superar la asignatura, a pesar de su moderada asistencia.

Continuando con el análisis de implicación del alumnado en el proyecto, se ha recopilado un indicador del grado de participación e implicación en las actividades del proyecto: el número de interacciones en la plataforma Tumblr (incluyendo en éstas las publicaciones, las veces que han pulsado "me gusta", el número de seguidores y el número de otros usuarios que los siguen). Esto queda reflejado en la Tabla 3 , que muestra un resumen de la participación de todos los grupos en la plataforma.

Tabla 3.

Resumen de la actividad por grupos de trabajo Tumblr.

\begin{tabular}{cccccl}
\hline $\begin{array}{c}\text { Grupo } \\
\text { Tumblr }\end{array}$ & $\begin{array}{c}\text { Publica- } \\
\text { ciones }\end{array}$ & $\begin{array}{c}\text { Me } \\
\text { gusta }\end{array}$ & Seguidores & Siguiendo & Observaciones \\
\hline 1 & 27 & 6 & 31 & 32 & $\begin{array}{l}\text { Han completado las actividades } \\
\text { además de realizar otro tipo de } \\
\text { publicaciones relacionadas con la } \\
\text { materia de la asignatura. }\end{array}$ \\
\hline 2 & 6 & - & - & - & $\begin{array}{l}\text { No han dedicado tiempo a la } \\
\text { realización de la actividad. }\end{array}$ \\
\hline 3 & 38 & 29 & 29 & 29 & $\begin{array}{l}\text { Han completado las actividades } \\
\text { además de realizar otro tipo de } \\
\text { publicaciones relacionadas con la } \\
\text { materia de la asignatura. }\end{array}$ \\
\hline 5 & 7 & 18 & 27 & 12 & $\begin{array}{l}\text { Han realizado un moderado número } \\
\text { de publicaciones, aunque todas } \\
\text { ellas de calidad. }\end{array}$ \\
\hline
\end{tabular}

Fuente: Elaboración propia 
Como se puede comprobar (Tabla 3), los grupos con mayor asistencia a la asignatura son también los más activos en la plataforma Tumblr (grupos 1 y 3). El grupo 1 ha sido algo más selectivo en la publicación y re-blogueo de la información. El grupo 3 , por el contrario, no es selectivo en los "me gusta", al igual que en las publicaciones, compartiendo publicaciones y fuentes de otros grupos. El grupo que presentó una mayor tasa de éxito (grupo 4) ha sido de los que menos ha participado aparentemente, ya que ha sido participativo con un moderado número de publicaciones, pero con gran implicación en ellas. Se han subido noticias comentadas con gran rigor, lo que ha demostrado dedicación y conocimiento. Ello se ha traducido en alto grado de éxito y rendimiento en la asignatura. El grupo 2 está formado por componentes repetidores de la asignatura que no han mostrado interés por la actividad. De hecho, solo han publicado dos noticias y han participado de las noticias de otros colgándolas en su muro. Por último, el grupo 5 se ha implicado aceptablemente en la publicación y seguimiento de sus compañeros/as. Es un grupo promedio en el grado de rigor científico y divulgación de contenidos. Esta variabilidad en el interés de la asignatura, como apuntan estudios anteriores, puede no reflejar una actitud negativa hacia el uso de redes sociales en el ámbito académico, sino más bien la percepción que tenga cada individuo hacia el valor del trabajo en grupo, siendo clave sus experiencias previas positivas en este tipo de trabajos (Almenara \& Díaz, 2014; Almenara, Osuna, Cejudo \& Cabrera, 2016). Estos autores además sugieren que el alumnado no presenta la misma familiaridad con redes sociales de tipo blog o wiki que con otras que involucran la publicación de fotos o vídeos. Asimismo, este estudio detecta posibles diferencias de género con este tipo de actividades que pueden ser resueltas promoviendo grupos mixtos.

Además del análisis descriptivo del grado de implicación por parte del alumnado en el proyecto de innovación a partir de su actividad en la plataforma Tumblr, se ha realizado una exploración gráfica de las calificaciones en las actividades del proyecto y de las calificaciones finales de la asignatura, mediante un análisis estadístico (Figura 5). El objetivo es intentar relacionar ambas calificaciones, si es posible.
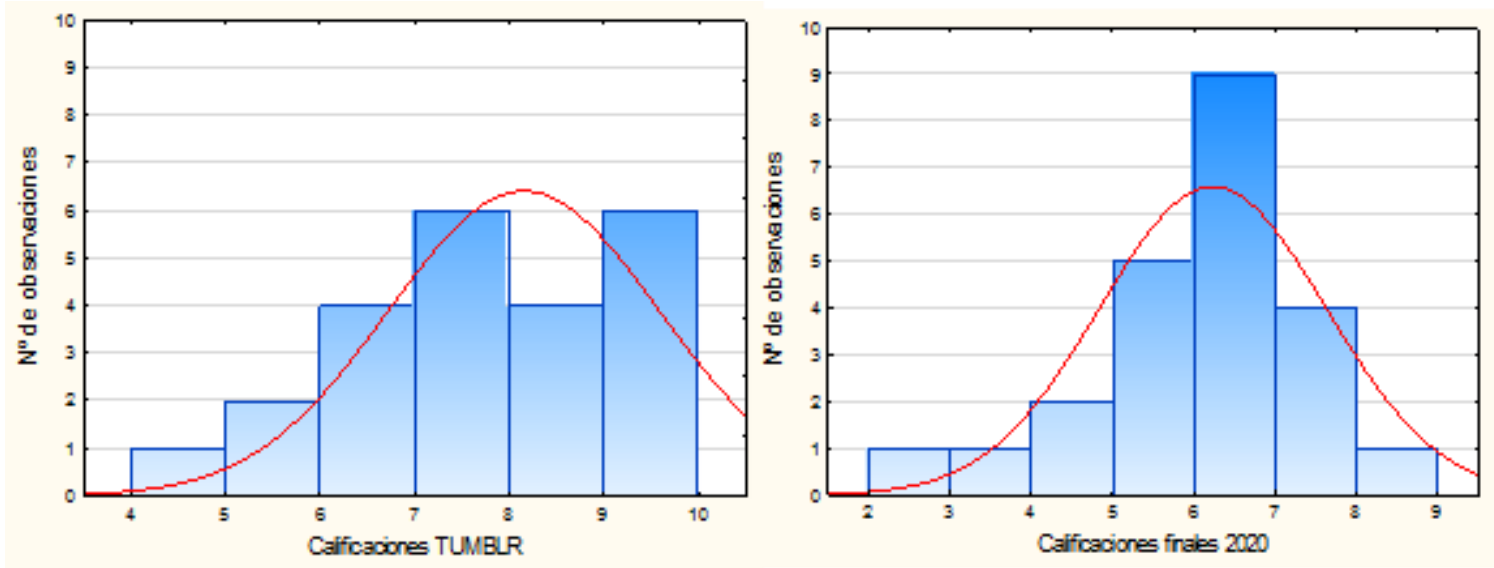

Figura 5. Histogramas de calificaciones en las actividades del proyecto (panel izquierdo) y calificaciones finales (panel derecho). Ajuste gaussiano en línea continua.

Fuente: Elaboración propia.

Como se puede observar, ambos histogramas presentan una distribución normal según el K-square test (Sheskin, 2020), con un p-valor de 0,56 en las actividades del proyecto y un $\mathrm{p}$-valor de 0,07 para las calificaciones finales. El rango de calificaciones es de 4-10 puntos en el caso de la actividad (nota media $8,2 \pm 1,4$ ) y de $2-9$ en la calificación global de la asignatura (nota media $6,2 \pm 1,4$ ). Se destacan un mayor número de observaciones/frecuencias con un rango de notas de 7-8 y 9-10 para las calificaciones en las actividades del proyecto Tumblr y un rango de 5-8 para las calificaciones finales de la asignatura. Además, destaca poco el número de alumnos/as 
ubicados/as en los extremos de la curva de distribución, lo que se traduce en un elevado número de personas con la calificación de aprobado.

Por otro lado, se hace indispensable una comparativa con el curso académico anterior, en términos relativos, dado el diferente número de alumnos/as por curso, en los que no se había implantado este tipo de proyecto. En este contexto, la Figura 6 muestra la evolución de las frecuencias relativas acumuladas en ambos cursos académicos (2018/19 y 2019/20) con respecto a las calificaciones finales obtenidas.

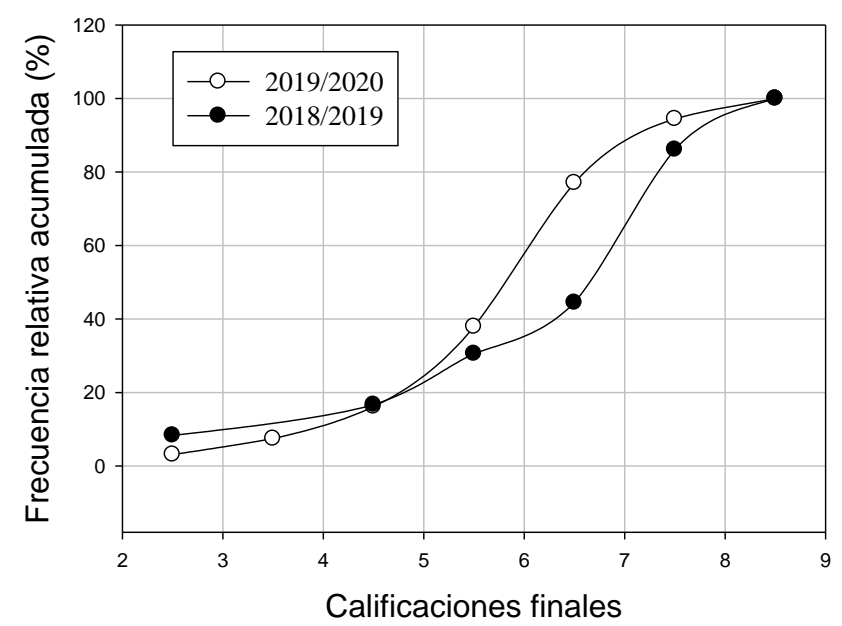

Figura 6. Evolución de las frecuencias relativas acumuladas en los cursos académicos 2018/19 y 2019/20 con respecto a las calificaciones finales.

Fuente: Elaboración propia.

Comparando la acumulación de ambas frecuencias relativas, no se observan diferencias significativas en las calificaciones inferiores a 5,0. Sin embargo, hay una desviación positiva en calificaciones en el rango de 5,0 a 7,5, lo que demuestra la validez del trabajo colaborativo de este proyecto. Las actividades del proyecto parecen aportar una herramienta de trabajo en competencias tales como la capacidad de gestionar la información y razonamiento crítico, que mejoran la evaluación final, entendiéndose ésta como el conjunto de competencias de habilidades, destrezas y conocimientos adquiridas por el/la alumno/a. Adicionalmente, la capacidad de análisis multidisciplinar de datos, índices e indicadores ambientales y cuantitativos a través de esta herramienta se trabaja de una forma innovadora y más llamativa que la resolución de la clásica colección de problemas.

Tras relacionar los resultados de la evaluación continua con los de la evaluación final, se observa una tendencia positiva entre los/as alumnos/as que más participan y los que alcanzaron un mayor rendimiento en la calificación final de la asignatura. Esta correlación indica que aquellos estudiantes que dedicaron una mayor atención y comprensión a las actividades relacionadas con el proyecto pudieron superar sin mayor problema la asignatura. Este resultado ha sido previamente descrito en estudios que han hecho uso de plataformas de microblogging en la educación superior (Fernández, Revuelta \& Sosa, 2012), que destacan que este cambio metodológico aumenta la motivación a través del aprendizaje activo, donde el alumnado es el protagonista, y logra la construcción de un discurso crítico y reflexivo, así como la adquisición de competencias como la síntesis comunicativa y la búsqueda, selección y tratamiento de la información. 
En el proyecto de innovación piloto se ha incluido una ponderación de solo un $5 \%$ en la calificación global, en previsión de que no se vea significativamente afectada si la actividad es un fracaso. En la Figura 7, se aprecia cómo los/as alumnos/as que obtuvieron calificaciones inferiores o iguales a 8 puntos en las actividades de Tumblr mostraban una tendencia proporcional en la calificación de la asignatura. La realización de la actividad proporciona información y competencias al estudiante en igual medida que éste/a le dedica atención al aprendizaje. Por el contrario, los/as alumnos/as con altas calificaciones no obtuvieron modificación significativa en la calificación. El primer grupo es aceptor de conocimiento, mientras que el segundo es el que lo genera y pone a disposición del grupo de forma pública. Este resultado sugiere que la calificación de esta actividad debería ser complementada con entrevistas individuales o bien con estrategias de coevaluación y autoevaluación (Jiménez, 2006), que permitan recompensar el esfuerzo de los individuos más activos de cada grupo, especialmente cuando ha resultado en la adquisición de competencias por parte de todo el grupo.

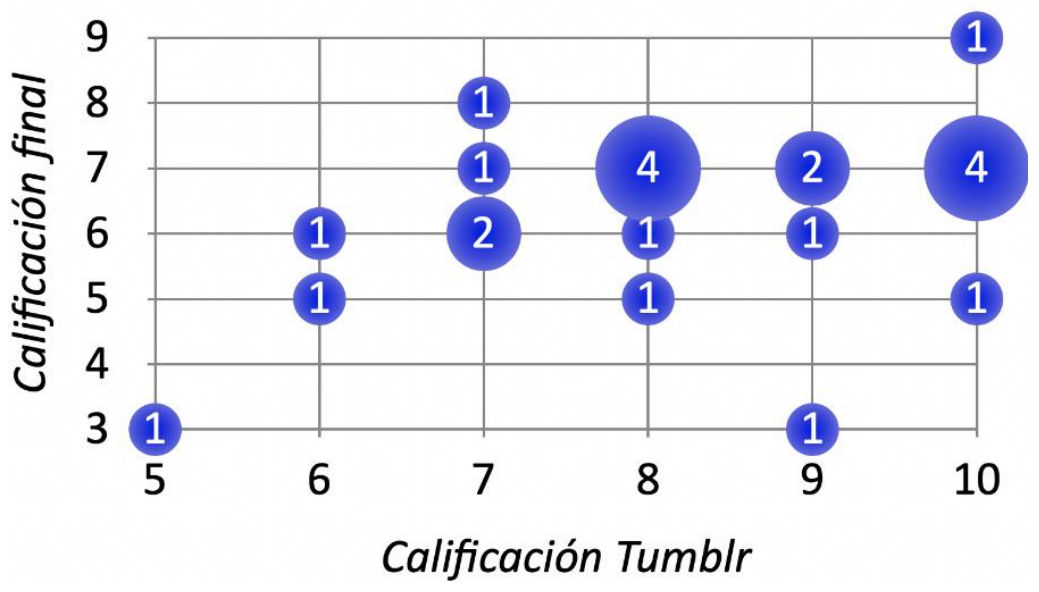

Figura 7. Relación entre las calificaciones obtenidas en las actividades del proyecto de innovación docente TUMBLR y las calificaciones finales de la asignatura para un/a mismo/a alumno/a (frecuencias indicadas en los círculos, con áreas proporcionales).

Fuente: Elaboración propia.

\section{CONCLUSIONES}

Como principal conclusión de la implantación de este proyecto de innovación se destaca la implicación del alumnado en su aprendizaje colaborativo. La creación de grupos, con distintos roles de sus componentes, da lugar a algunas publicaciones con alto grado de creatividad. Esto redunda en una clara recompensa, tanto en el desarrollo de las competencias relacionadas con el proyecto, como en una mayor posibilidad de superar la asignatura. La relación entre la evaluación continua de esta actividad y las calificaciones finales de la asignatura ha revelado que el alumnado con calificaciones intermedias es el que ha mostrado una mejora más significativa en su rendimiento académico. No obstante, se ha detectado que la asistencia a la actividad, por sí misma, no representa un indicador fiable del desarrollo de las competencias de la asignatura.

Se considera, por tanto, que este proyecto se puede plantear como una actividad de formación valiosa para el aprendizaje continuo y evaluación, complementaria a las ya existentes en la asignatura, y que permite seguir una estrategia de diversificación a la hora de evaluar de forma precisa el rendimiento del alumnado, sobre todo en la comunicación de información científico-tecnológica mostrando los frutos de su aprendizaje, ofreciendo una estrategia de motivación en grupo a la vez que permite cierta flexibilidad horaria y temática. Queda por explorar, como línea de trabajo futura, el efecto de complementar la calificación de esta actividad con entrevistas individuales 
o estrategias de coevaluación, que permitan recompensar el esfuerzo de los individuos más activos de cada grupo.

La principal dificultad ha sido el cribado de información, en la que se pretendía que desarrollaran un aspecto crítico con respecto a la información que circula en la red, razonando de forma científica su veracidad. Esta actividad ha demostrado ser sensiblemente la más costosa para el alumnado y, por tanto, la que menos interés ha despertado en el mismo. Por ello, es necesario un seguimiento más cercano de las actividades de cribado de información, en interacción con el profesorado y en conjunto con todos los grupos participantes. Esto permitirá guiar al alumnado en el tipo de preguntas que deben hacerse, fomentando así el razonamiento crítico que permita analizar la veracidad de las fuentes consultadas.

En cuanto a la adquisición de competencias de conocimiento, se ha demostrado que los/as estudiantes han preferido buscar fuentes de información con noticias, textos o imágenes en los que ellos/as tuvieran nociones de la información y, en ocasiones, no enfocada a la materia de la asignatura. Este hecho se podría mejorar adaptando y acotando inicialmente las fuentes de información para su uso por parte del alumnado, ofreciendo unas orientaciones generales y que en todo momento permita encontrar la compatibilidad de sus intereses con respecto a la asignatura.

\section{AGRADECIMIENTOS}

Los autores desean agradecer a la Universidad de Córdoba por haber financiado este Proyecto de Innovación Docente (2019-2-2004) a través del Plan de Innovación y Buenas Prácticas Docentes del curso 2018/2019 y el Plan Propio de Investigación 2019 (submodalidad 2.4.).

\section{REFERENCIAS}

Almenara, J.C., Osuna, J.B., Cejudo, M.D.C.L., \& Cabrera, C.Y. (2016). Redes sociales y Tecnologías de la Información y la Comunicación en Educación: aprendizaje colaborativo, diferencias de género, edad y preferencias. Revista de Educación a Distancia, (51). Recuperado de https://bit.ly/2ZfID30

Almenara, J.C., \& Díaz, V.M. (2014). Posibilidades educativas de las redes sociales y el trabajo en grupo.: Percepciones de los alumnos universitarios. Comunicar: Revista científica iberoamericana de comunicación y educación, (42), 165-172. DOI: http://dx.doi.org/10.3916/C42-2014-16

Bourlai, E.E. (2018). 'Comments in Tags, Please!': Tagging practices on Tumblr. Discourse, context \& media, 22, 46-56. DOI: https://doi.org/10.1016/j.dcm.2017.08.003

Chang, Y., Tang, L., Inagaki, Y., \& Liu, Y., (2014). What is tumblr: A statistical overview and comparison. ACM SIGKDD explorations newsletter, 16(1), 21-29. DOI: https://doi.org/10.1145/2674026.2674030

De Haro, J.J. (2010). Redes sociales en educación. Educar para la comunicación y la cooperación social, 27, 203-216. Recuperado de https://bit.ly/2CJlcqZ

Deller, R. (2015). Simblr famous and SimSecret infamous: Performance, community norms, and shaming among fans of the Sims. Transformative Works and Cultures, 18. DOI: https://doi.org/10.3983/twc.2015.0615

Fernández, M.R., Revuelta, F.I., \& Sosa, M.J. (2012). Redes sociales y microblogging: innovación didáctica en la formación superior. RELATEC. Recuperado de https://bit.ly/2VqGP5W 
Gómez, M., Roses, S., \& Farias, P. (2012). El uso académico de las redes sociales en universitarios. Comunicar, 19(38), 131-138. DOI: https://doi.org/10.3916/C382011-03-04

Imbernón, F., Silva, P., \& Guzmán, C. (2011). Competencias en los procesos de enseñanza-aprendizaje virtual y semipresencial. Comunicar, 18(36), 107-114. DOI: https://doi.org/10.3916/C36-2011-03-01

Jiménez, G. (2006). Obtención de notas individuales a partir de una nota de grupo mediante una evaluación cooperativa. Revista lberoamericana de Educación (OEI), 38 (5). Recuperado de https://bit.ly/2BIStCr

Martínez, J.D.J.C. (2014). Las redes sociales en la educación superior. Educación y Desarrollo Social, 8(1), 102-117. DOI: https://doi.org/10.18359/reds.586

Martínez, J.G., Fortuño, M.L., \& Vidal, C.E. (2016). Las redes sociales y la educación superior: las actitudes de los estudiantes universitarios hacia el uso educativo de las redes sociales, de nuevo a examen. Education in the Knowledge Society, 17(2), 21-38. DOI: http://dx.doi.org/10.14201/eks20161722138

Rubio-Millares, R. (2019). Expandir el aula con Tumblr. Instituto Nacional de Tecnologías Educativas y de Formación del Profesorado, 27, 1-8. Recuperado de https://bit.ly/3bh9hOy

Santana, P. J., Jiménez, F., Pintor, P., \& Hernández, V. M. (2017). Cómo implicar al alumnado en la evaluación formativa. El valor del feedback. Servicio de publicaciones de la Universidad de La Laguna. Recuperado de: https://bit.ly/3eBy4xz

Sheskin, D. J. (2020). Handbook of parametric and nonparametric statistical procedures (5th ed.). CRC Press. DOI: https://doi.org/10.1201/9780429186196

TAR (2019). Tratamiento de Aguas Residuales, Grado de Ciencias Ambientales de la Universidad de Córdoba. Recuperado de https://bit.ly/3i475Nn

Tumblr (2020). Acerca de Tumblr. Recuperado de https://bit.ly/3dNf91L

van Dijck, J. (2013). The Culture of Connectivity. A Critical History of Social Media Oxford University Press, Oxford. DOI: http://dx.doi.org/10.7146/mediekultur.v30i56.16314

Vidal, C.E., Martínez, J.G., Fortuño, M.L., \& Cervera, M.G. (2011). Actitudes y expectativas del uso educativo de las redes sociales en los alumnos universitarios. RUSC. Universities and Knowledge Society Journal, 8(1), 171185. DOI: http://dx.doi.org/10.7238/rusc.v8i1.839

Yunus, M.M., \& Salehi, H. (2012). Tumblr as a medium to improve students' writing skills. Journal of Applied Sciences Research, 8(1), 383-389. Recuperado de https://bit.ly/3g740Kx 